\title{
SHIELDING MEASUREMENTS OF THE SPACE SHUTTLE (PART II - TESTING THE NASA ENDEAVOUR)
}

\author{
Diane Kempf \\ Buzz Brezinski \\ Naval Air Warfare Center \\ Patuxent River, Maryland
}

\author{
Robert Scully \\ NASA Johnson Space Center \\ Houston, Texas
}

\begin{abstract}
Testing was conducted on the NASA Space Shuttle Orbiter, Endeavour, to determine the high frequency shielding capability of three major cavities; the flight deck, the mid-deck/crew quarters and the payload bay. Testing was performed using highly modified reverberation techniques. The hangar at the Shuttle Landing Facility at Kennedy Space Center served as the "reverberation chamber" for the spacecraft. An account of the test method is presented in detail, along with setup diagrams, photos, and graphs of the shielding measurements.
\end{abstract}

\section{Background}

The Naval Air Warfare Center (NAWC) at Pax River was part of a team effort with the National Institute of Standards and Technology (NIST) to perform a shielding test of the NASA Space Shuttle Orbiter "Endeavour" in the frequency range of $30 \mathrm{MHz}$ to 18 GHz. Pax River was responsible for the $4 \mathrm{GHz}$ to 18 $\mathrm{GHz}$ frequency range. Testing was conducted on the Orbiter to determine the high frequency shielding capability of three major cavities; the flight deck, the crew quarters, and the payload bay. In the usual NAWC EMI tradition, testing was performed using highly modified reverberation techniques. This presentation gives an account of the test method for the Space Shuttle in detail, along with setup diagrams, photos of the testing of the Space Shuttle, and graphs of the shielding measurements. Reverberant technology has been demonstrated to be statistically highly repeatable and uniform over various reverberant environments. This "Endeavour" provided NASA with much needed shielding information that also demonstrated to be statistically highly repeatable and uniform throughout the reverberant test environment.

The only comprehensive shielding evaluation conducted on the National Aeronautics and Space Administration (NASA) Space Shuttle Orbiter was in the early 1980 's, but has been highly scrutinized. As a result of the Columbia accident, NASA made plans to implement much higher power tracking radars to identify potential debris and subsequent tile system damage during night launch. NASA leadership recognized that new approaches to EMI are imperative and have demonstrated their commitment to change with a recent program to examine the shielding of the Space Shuttle Orbiter. This paper discusses the 4.2 to $18 \mathrm{GHz}$ frequency range that NAWC Pax River was responsible for in a joint venture with NASA and the National Institute of Standards and Technology (NIST). NIST was primarily responsible for the lower frequencies, but also provided consultation for the higher frequency range. The methodology is a progression of ideas developed by NAWC, the Naval Surface Warfare Center (NSWC), NIST, and NASA. (See references 1, 2, 3, and 4). This "endeavour" is a case study of a truly unique EMI after-the-fact avionics-related evaluation.

\section{The Equipment Under Test}

The Space Shuttle Orbiter, specifically the Endeavour, is one of NASA's reusable space vehicles and is approximately 122 feet long, 57 feet high (on runway) and has a wingspan of 78 feet. The Orbiter consists of several cavities not limited to; the flight deck/aft flight deck, mid deck/crew compartment, payload bay, and avionics bays. The flight deck contains six pressure windshields, two overhead windows, and two rear-viewing payload bay windows. The crew compartment is a pressurized working, living, and stowage compartment consisting of 2525 cubic feet in the forward portion of the Orbiter. The payload bay is approximately 60 feet long and 15 feet in diameter. The doors to the payload bay are on the top portion of the fuselage and are 60 feet long. The Orbiter was not in a true flight configuration, but all altered/missing components were examined and it was determined that the test configuration would yield a worst case.

\section{Test Methodology}

Testing was performed using a state of the art version of reverberation methods. Traditionally, a reverberant shielding effectiveness test is performed using "nested reverberation chambers." The field in each chamber is "stirred" using an electrically large metallic tuner that is capable of suitable displacement at the lower 
frequency range of the test. Statistically, this "stirring" produces a very uniform field over a revolution of the tuner. Since it is physically impractical to construct and locate a tuner that would be large enough to be effective to use in a chamber of the size needed, this test uses several antennas placed around the aircraft to provide spatial sampling instead of displacement sampling (reference 3). Another array of antennas is placed inside the aircraft that are moved to various locations for internal spatial sampling. Frequency averaging, or electronic stirring, is used to simulate the physical boundary movement of the RF standing wave patterns and augment the statistical sampling in the cavity (reference 2). As in the traditional methods, the received levels are compared to those received when the aircraft is not present (the reference measurements). The difference is the shielding effectiveness of the aircraft. A big advantage of this method is that it avoids the inaccuracies associated with conventional direct radiation methods caused by: standing wave patterns, radiation aspect angle, and antenna factor errors. These usually render very inaccurate and unrepeatable measurements. Reverberant technology has been demonstrated to be statistically highly repeatable and uniform over various reverberant environments.

\section{Test Site}

\section{Space Port Florida-The Final Space}

There was necessarily a concern about where the "Endeavour" might take place. Originally, a huge metallic tent was proposed around the Orbiter in the Vehicle Assembly Building. However, NASA skillfully negotiated the use of Spaceport Florida, called the Shuttle Landing Facility (SLF) Hangar by NASA personnel. The SLF is a state owned facility on the Kennedy Space Center complex already in use by hurricane displaced NASA personnel. This hangar measures 250 feet by 201 feet, with a curved ceiling, 65 feet high at the center. The walls and ceiling are constructed of metal and covered with foil backed fiberglass insulation and the floor is concrete. Equipment and personnel were arranged along each side of the hangar for other NASA work and they co-existed with the test personnel through test progression. Therefore, the test area was limited to approximately the middle half of the hangar. It was befitting that the hangar that had housed the Columbia wreckage investigation would provide a test platform for the shielding test to assist with Return to Flight night launch.

\section{Evaluation of the SLF "Reverberation Chamber"}

During January 18 through 21, 2005, the Pax River EMI Lab, NASA, and NIST performed measurements in the SLF Hangar in order to determine its suitability for the shielding effectiveness test of the Orbiter. NAWC performed brief measurements with limited equipment at a few spot frequencies. NASA supplied the equipment and test personnel to operate the equipment according to the NAWC Test Plan. Of main concern was to determine its uniformity, and to obtain a rough measure of its cavity insertion loss.

Measurements were performed at 4, 8, 12, and 18 $\mathrm{GHz}$ at four locations in the hangar without the Orbiter present. The field uniformity was typically $\pm 2.5 \mathrm{~dB}$, with a worst case of $\pm 4 \mathrm{~dB}$. Since it was simpler to perform peak measurements with the instrumentation used, the average received power was calculated using NIST published peak to average ratios for a reverberation chamber (reference 5). The hangar insertion losses are as shown in Table 1. Note that these are only approximate, based on the uncertainties involved with calculating an average from peak measurements with a limited amount of data.

Table 1. SLF Insertion Loss

\begin{tabular}{|c|c|}
\hline Frequency & Insertion loss \\
\hline \hline $4 \mathrm{GHz}$ & $66 \mathrm{~dB}$ \\
\hline $8 \mathrm{GHz}$ & $75 \mathrm{~dB}$ \\
\hline $12 \mathrm{GHz}$ & $81 \mathrm{~dB}$ \\
\hline $18 \mathrm{GHz}$ & $86 \mathrm{~dB}$ \\
\hline
\end{tabular}

\section{Orbiter Shielding Effectiveness Test}

\section{Equipment Layout}

A layout of the Orbiter Shielding Test equipment is provided in Figure 1. Two computer test systems were used. Although the systems were identical and 100\% interchangeable, one was designated the control and the other the processing station. These were linked together via link network to share files. The horn antennas were oriented randomly, but logically to assure good statistical data. Optical links were used to control the two receive towers, which consisted of two spectrum analyzers, two preamplifiers, two antennas, an uninterruptible power supply, an optical link and cabling. A third spectrum analyzer was included on one tower to monitor ambients. Separate $200 \mathrm{ft}$ power cords fed each of the receive towers, the processing station, and the transmit rack. This allowed free independent movement of all components. Each tower was fed with an independent 60-foot optical control link. A separate optical link was provided to the transmit rack, containing the synthesizer, amplifier, and RF switch. 


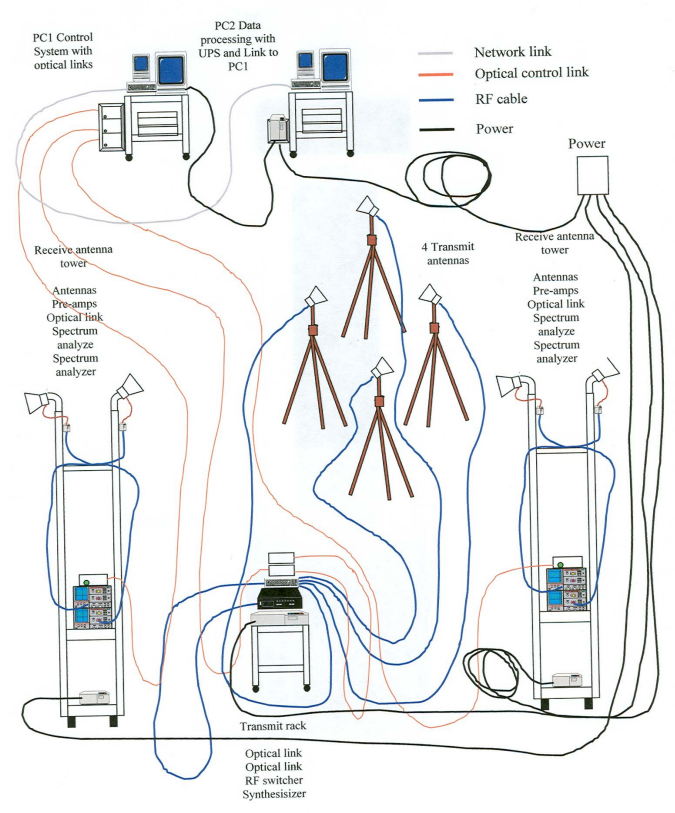

Figure 1. Shielding Equipment Layout

Of course, this transmit system was only on a cart when conducting reference measurements outside the Orbiter. When inside the Orbiter, it was split to facilitate better movement to the various test locations. The transmit antennas were taken off the tripods and mounted to a single antenna stand in the Orbiter. All cables in the Orbiter installation used prodigious quantities of ferrites to prevent RF feedback, including power cord penetration into the Orbiter and between cavities. Power in the Orbiter was filtered prior to feeding equipments. Figure 2 shows a typical set up of control/processing relative to receive towers. The two towers were typically close enough to the processing stations to provide for quick observations of signals relative to noise floor.



Figure 2. Typical Layout of Control and Processing System Relative to Receive Tower

\section{Reference Measurements}

Reference measurements are essentially a measurement of the insertion loss of the reverberant cavity that the test item is placed in. A measured power is transmitted into the cavity, the SLF hangar in this case, and the power received by the receive antennas is recorded for each frequency. This is the baseline that the Orbiter measurements are compared to, after both sets of measurements are normalized. The difference between the reference measurements and the measurements performed with the transmit antennas inside the Orbiter is then the shielding effectiveness of the Orbiter.

Reference measurements were performed with the Orbiter in the reverberation structure. The reference runs consisted of 8 data sets; two transmit locations, with four receive locations for each, covering the 4 quadrants of the Orbiter. This resulted in 16 files $(4$ transmit X 4 receive) at each of 8 locations with a 20 sample frequency stir step. This equals 2560 samples/frequency step. The receive antennas were typically pointed away from the Orbiter to allow for maximum reverberation of the transmit signal before it arrived at the receive antennas. This configuration avoided direct and first reflection coupling of the transmit and receive antennas, and the possible excessive absorption due to the spacecraft. Also, care was taken to avoid any symmetry in the receive antenna locations. The transmit antennas are shown in Figure 3. The receive antenna locations are shown in Figure 4.

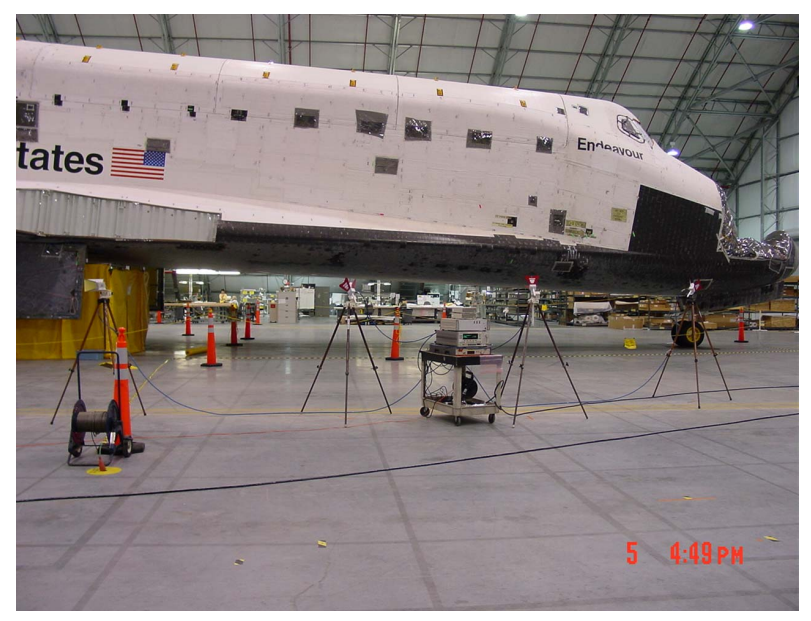

Figure 3. Reference Measurements - Transmit Antennas 


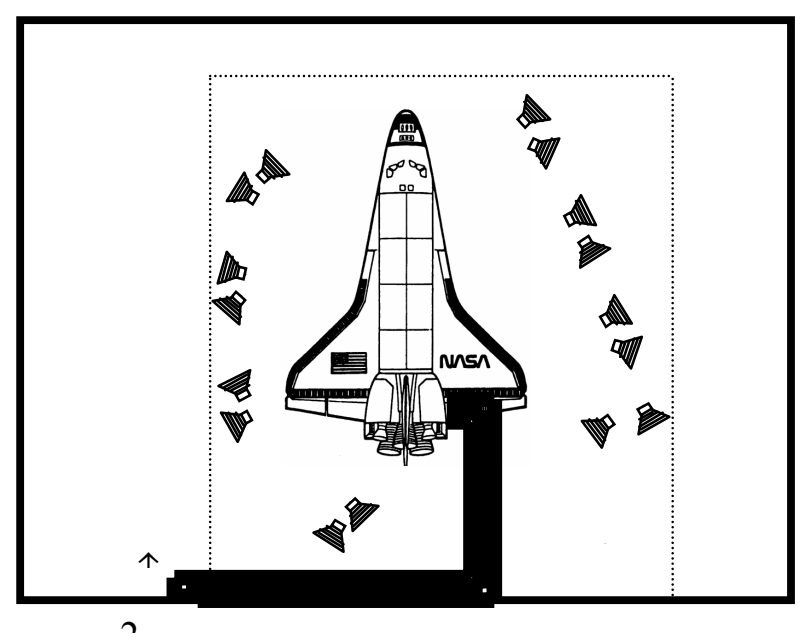

Figure 4. Receive Antenna Locations

SLF Hangar Insertion Loss or "Loss in Space"

An average of all of the normalized data gathered during the reference measurements is essentially the average insertion loss of the SLF Hangar. This was used as the reference to which all of the Orbiter measurements were compared to, in order to calculate the shielding effectiveness. This is shown in Figure 5. The bounds for the 8 individual test positions that make up this data are provided in Figure 6. This is basically the uniformity of the chamber. As with other enclosure evaluations using this methodology, the cumulative probability of the reference measurements was compared to an ideal $\mathrm{Chi}^{2}$ distribution. This is provided in Figure 7. Note that there is some departure from the ideal, but it is much better than the NAWC Shielded Hangar, where excellent results had previously been achieved.

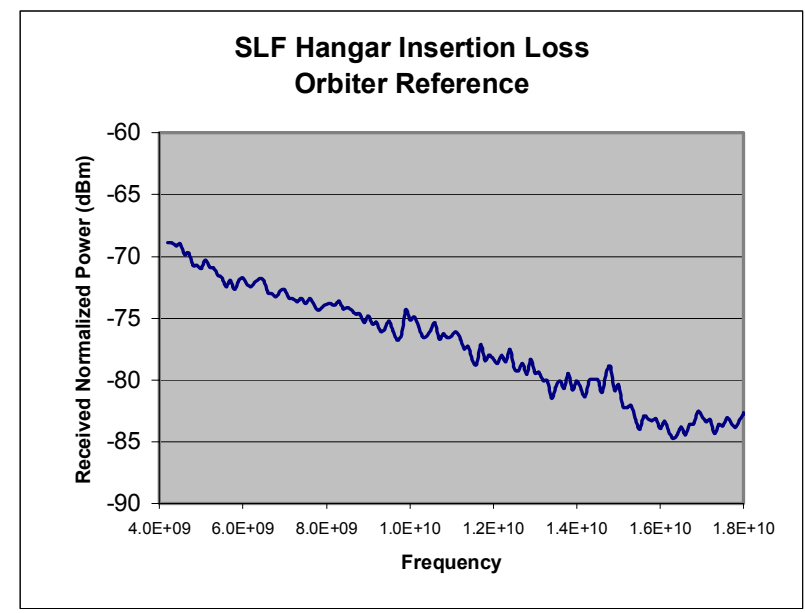

Figure 5. SLF Hangar Insertion Loss

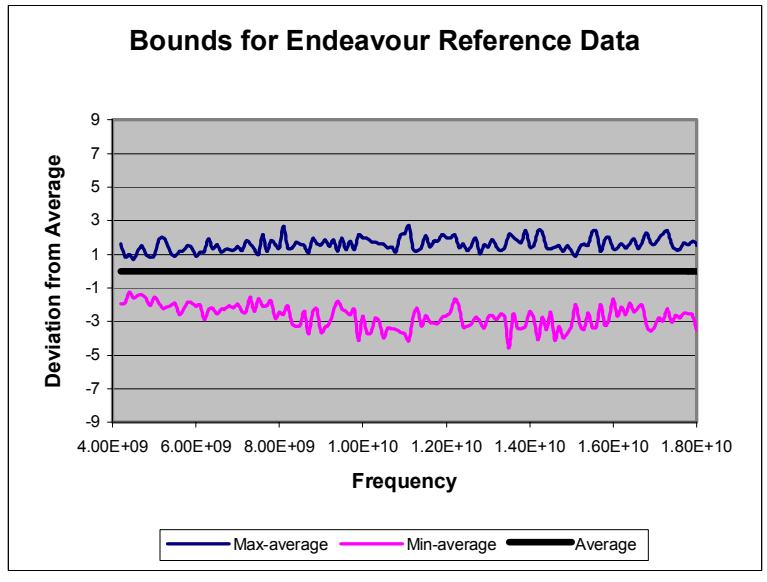

Figure 6. Bounds for Reference Data

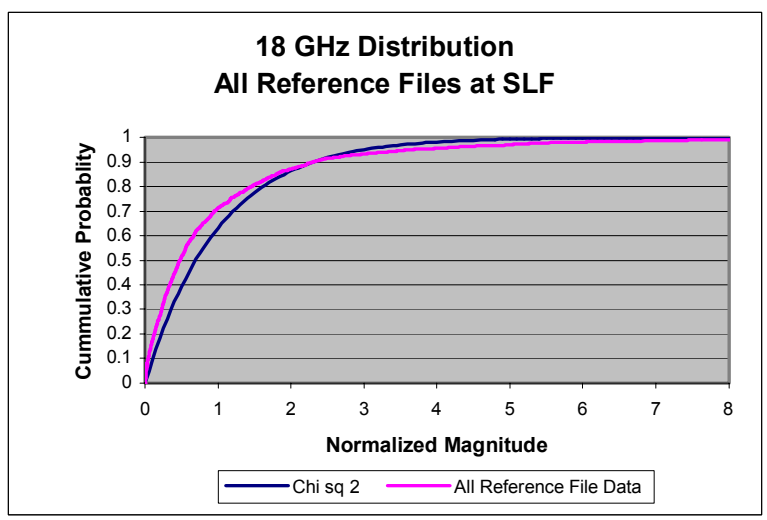

Figure 7. 18 GHz Cumulative Probability Distribution in the SLF Hangar

The Test "Endeavour"

Test Set-up

For the Orbiter shielding test, equipment was distributed in two areas as shown in Figure 8.

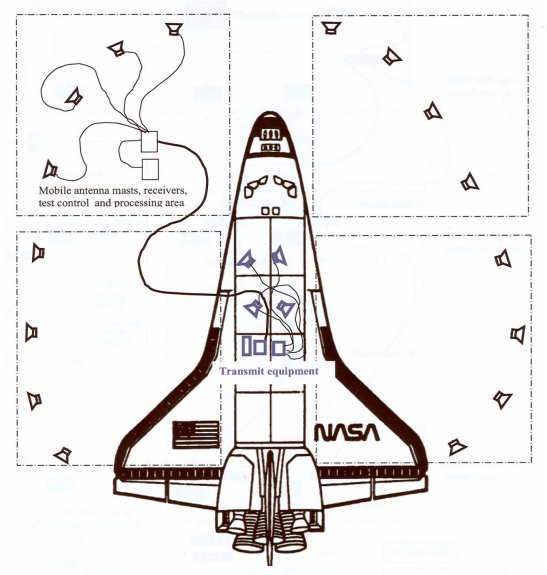

Figure 8. Equipment Setup Showing 4 Receive Test Quadrants (one at a time) 
Outside of the Orbiter were two moveable receive towers. A fiber optic link provided communication with the test computers. Four transmit antennas were located in the Orbiter cavity being tested. Figure 9 shows the approximate locations of the transmit antennas to test the three cavities. Internal antennas were asymmetrically placed about each cavity. Four antenna positions were used in each of the flight deck, and crew compartments, resulting in 16 files at each of 4 receive locations, using 20 stir frequency samples for a total of 1280 samples at each frequency step. Six transmit antenna positions were used in the payload bay section, three at a time for a total of 1920 samples at each frequency step.

Antenna Positions

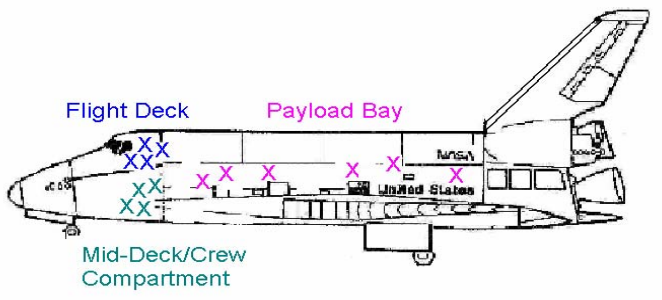

Figure 9. Transmit Antenna Locations

\section{Results of the Orbiter Shielding Effectiveness Measurements}

The shielding results provided below demonstrate relatively flat characteristics, unlike traditional methods, which display very large swings in data from frequency to frequency. Note that this is not necessarily a result of the methodology, but this method does tend to provide smoother, more consistent results. However, resonant effects are easily identified using this methodology. These effects are normally only encountered in very tight shielding situations with highly tuned slots. Typically, broad apertures emit a range of frequencies consistently, as is the case with the Orbiter. Although the payload bay would appear to have highly tuned slots at the seams of the doors, they are most likely much longer than the wavelengths of the frequencies involved, so they tend to attenuate consistently across the 4.2 to $18 \mathrm{GHz}$ band.

The results are presented as relative numbers, since the distribution of the actual shielding data is limited to NASA and its support contractors.

NIST data overlapped that of NAWC in the frequency range of 4.2 to $6 \mathrm{GHz}$. Interestingly, even with two completely different test methodologies, there was consistently good agreement between like sets of data in insertion loss, uniformity, and shielding values.

\section{Flight Deck}

The average shielding results for the flight deck are shown in Figure 10. This shows the average shielding measured with all four transmit antennas. The range of the shielding was approximately $4 \mathrm{~dB}$ throughout the 4.2 to $18 \mathrm{GHz}$ frequency range. Figure 10 also shows the shielding measured using transmit antenna \#1 only, which was aimed directly out the flight deck window. In this case, the shielding was approximately $3 \mathrm{~dB}$ lower than when all four transmit antennas were averaged together. This represents worst case shielding for the flight deck.

\section{Mid-Deck}

The shielding of the Orbiter in the mid-deck in the 4.2 to $18 \mathrm{GHz}$ frequency range is shown in Figure 10 . The range of the shielding was approximately $4 \mathrm{~dB}$ throughout the 4.2 to $18 \mathrm{GHz}$ frequency range.

\section{Payload Bay}

While collecting data for the payload bay, the preliminary shielding values were somewhat higher than expected. Due to the large longitudinal seam along the payload bay doors that was not designed to provide RF shielding, it was expected that the shielding of the payload bay would be very poor. It was suspected that the radiation leakage pattern of the payload bay door seam might have been aimed primarily up towards the ceiling. Therefore, one receive antenna was aimed up at the peak of the ceiling in an attempt to catch the first bounce of the signals leaking out the seam of the payload bay doors. This only had an effect of about 2-3 dB on the shielding values. Other antenna positions were used in an attempt to determine if any particular payload bay "antenna" and hangar shape interaction was going on, but none of these additional measurements indicated anything unusual. Extensive equipment checks were performed in order to eliminate any possibility of error. It is uncertain at this time exactly why the payload bay was found to have such excellent shielding. Detailed analysis of the data has led us to some theories but without further testing, modeling, and simulations, it is premature to present them in this report. In any case, any skewing of the measurements due to unusual spacecraft/hangar interactions affecting the reference measurements and the payload bay shielding measurements differently appears to have an effect on the payload bay shielding values of only a few $\mathrm{dB}$. The shielding measurements for the payload bay are shown in Figure 10. 


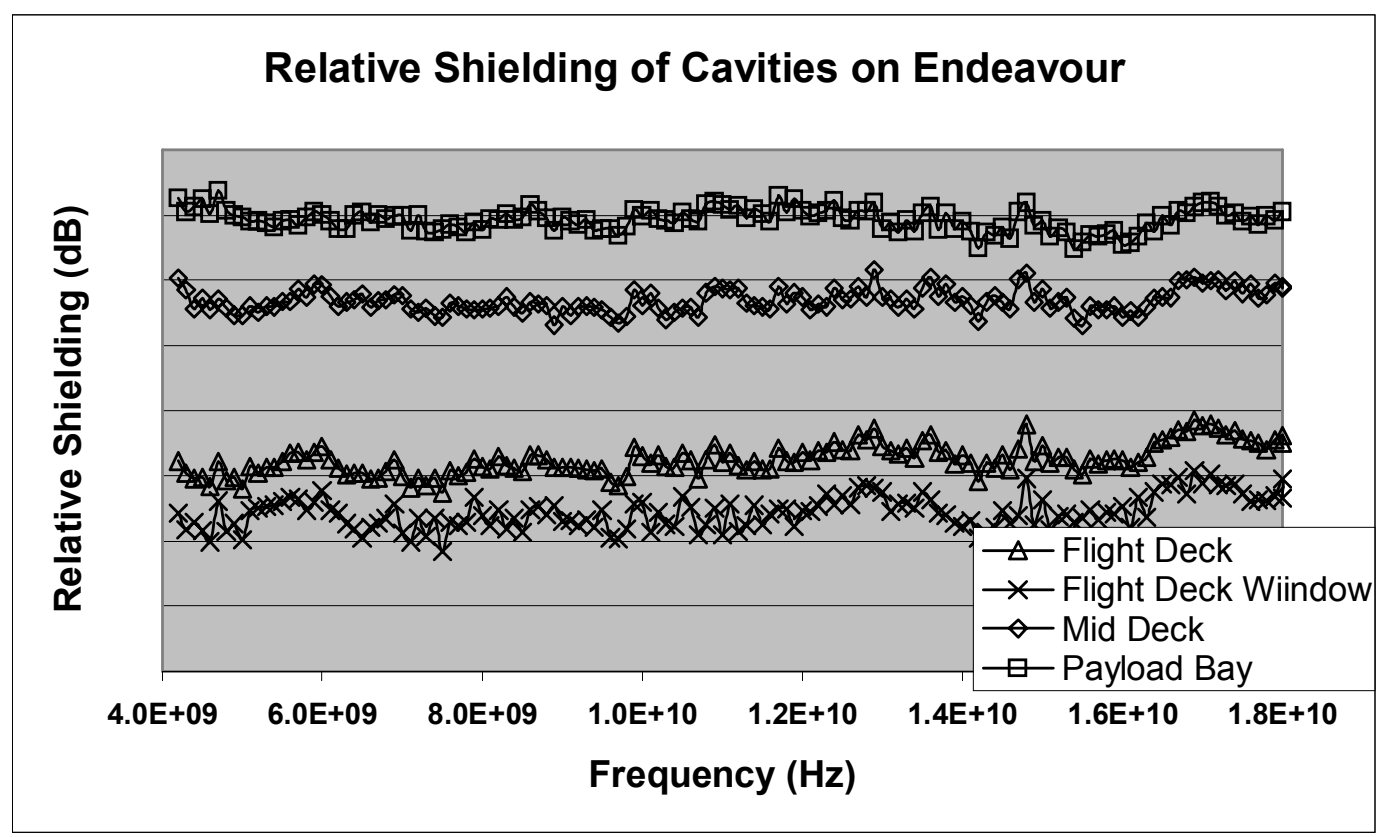

Figure 10. Relative Orbiter Shielding

\section{Conclusions}

New methodologies are needed to conduct more realistic EMI evaluations of avionics equipments. EMI spaces and practices do not necessarily need to conform to the usual paradigms. Teamwork and innovation paid off big dividends in achieving success with this large "Endeavour".

Testing in the large SLF Hangar and the Pax River Shielded Hangar has shown that there are test phenomena unique to very large reverberation chambers that need further investigation.

\section{Acknowledgements}

The PAX River shielding test team would like to thank the entire NIST test team: Bob Johnk, David Navotny, Chris Grosvener, Nino Canales, and Dennis Camell for their insights, suggestions, and expertise. It was a pleasure working with them all. Also of NIST, we especially acknowledge Galen Koepke. With his vast experience and knowledge of reverberation methods, Galen provided valuable advice and was an excellent liaison between the NIST and Pax test teams, using two very different test methods to measure the shielding of the Orbiter. All of the folks at NASA, too numerous to name here, who coordinated the effort, supported the Orbiter, and provided assistance when needed, are all greatly appreciated. We heartily thank our Team who fully supported the "Endeavour" by getting equipment together, organizing and delivering that equipment and filling the gaps in workload back home during the "Endeavour". And last, but certainly not least, a big thanks to Bob Scully of NASA, a true leader who facilitated this Endeavour in spite of all the odds. We are grateful to be part of such a successful, interesting, project.

\section{References}

1. Kempf, Diane R., Kenneth “Buzz” Brezinski, October 2001, Using Reverberation Techniques for Testing an Intercommunications System on a Navy Aircraft (2001: A Test Lunacy), 2001 IEEE Digital Avionics Conference

2. Grosvenor, Chriss. A., Robert T. Johnk, David R. Novotny, Nino Canales, Claude M. Weil, Jason Veneman, January 2003, A Two-Phase Airframe Shielding Performance Study Using Ultra-wideband Measurements Systems, NISTIR 6622, National Institute of Standards and Technology

3. Hatfield, Michael, April 2003, "In-Situ Shielding Measurements", Reverberation Chamber, Anechoic Chamber, OATS, and GTEM User's Meeting

4. Kempf, Diane R., Kenneth "Buzz" Brezinski, September 2002, Shielding Effectiveness Testing of the Learjet 45, NAWC AD EMI Report

5. Ladbury, John M.; Galen H. Koepke, June 1999, Reverberation Chamber Relationships: Corrections and Improvements or Three Wrongs Can (Almost) Make a Right, Reverberation Chamber, Anechoic Chamber, and OATS User's Meeting 